\title{
Exploring Environments by Hand or Foot: Time-Based Heuristics for Encoding Distance in Movement Space
}

\author{
Susan J. Lederman \\ Queen's University at Kingston, Ontario, Canada \\ Roberta L. Klatzky \\ University of California at Santa Barbara \\ April Collins and Jackie Wardell \\ Queen's University at Kingston, Ontario, Canada
}

\begin{abstract}
In Experiment I, blindfolded observers judged (a) the distance of pathways felt by hand and (b) the straight-line distance between pathway endpoints inferred from such exploration. In Experiment 2, blindfolded observers made corresponding estimates after traversing similar pathways on foot. Pathways were explored under three different speeds. Under both manipulatory and ambulatory exploration, there was substantial length distortion of inferred distance: The straight-line distance was increasingly overestimated with increases in the length of the explored pathway. With manipulatory exploration, slower movements increased length distortion, but duration effects proved secondary to effects of spatial extent. For ambulatory exploration, no duration effects were obtained. Observers used time-independent heuristics, that is, a footstep metric for estimating the pathway actually travelled and a spatial imaging strategy for estimating the inferred line between pathway endpoints. The studies establish length distortion as a general phenomenon in movement space and identify its major causes as spatial rather than temporal.
\end{abstract}

Substantial research in visual perception and cognition has investigated how an observer achieves a representation of the layout of objects in space and how that representation departs from veridicality (e.g., Gogel, 1978; Hirtle \& Jonides, 1985; Moar \& Bower, 1983; and see later discussion). In contrast, we know little about the representation of spatial layout by observers who are denied the use of sight.

Theories of spatial representation frequently distinguish between large- and small-scale space. Note that size and scale are not considered synonymous. For example, according to Siegel (1981), distinctions in size (e.g., large, small) involve comparisons of space along some physical metric, for example, centimeters or meters. In contrast, distinctions in scale involve comparisons of the perceptual and motor mechanisms used.

Acredolo (1981) articulated two major features of a largescale environment, based on a discussion by Ittelson (1973). First, a large-scale space encloses an observer; a small-scale space does not. Second, a large-scale environment is typically explored from multiple vantage points, and to this extent in-

This study is derived from a joint research program by Susan J. Lederman and Roberta L. Klatzky.

The work is supported by Natural Sciences and Engineering Research Council of Canada Grant A9854 to Susan J. Lederman and National Science Foundation Grant BNS-84-21340 to Roberta L. Klatzky. Experiments 1 and 2 formed undergraduate honors psychology theses by April Collins and Jackie Wardell, respectively, supervised by Susan J. Lederman.

Correspondence concerning this article should be addressed to Susan J. Lederman, Department of Psychology, Queen's University, Kingston, Ontario, Canada K7L 3N6; or to Roberta L. Klatzky, Department of Psychology, University of California at Santa Barbara, Santa Barbara, California 93106. volves integration of information over time. The first feature is the more critical because small-scale spaces may also require viewing from multiple vantage points. This same point has been emphasized by Gibson (e.g., 1950), who noted that few environments, regardless of size, are in fact encoded in a single glance. Most are actually explored over time by using head or eye movements or both.

Recognizing the validity of Gibson's argument, Garling, Book, and Lindberg (1985) offered a third criterion that is also based on typical rather than necessary differences in perceptuomotor mechanisms. They suggested that the presence of wholebody locomotion (in addition to head or eye movements, or both) usually indicates a large-scale environment, and its absence a small-scale environment. This third criterion thus differentiates scale on the basis of the sensorimotor systems typically used.

Note that these distinctions of space are based on the use of sight. There may be other distinctions that are more critical when considering movement space. By this term, we mean space that is explored without vision (e.g., by a blindfolded or blind observer) and is apprehended haptically, entirely by exploratory movements of the arm or leg systems. Thus, the primary basis for differentiating movement spaces is whether the exploring instrument is the arm system (i.e., fingers/hands/arms) or the leg system (i.e., toes/feet/legs). This distinction is related to the scale distinction in visual space, in that small-scale space would be explored through movements of the arms, hands, and fingers, and large-scale space through ambulation. We refer to smallscale layouts explored with the arm system as manipulatory spaces, and large-scale layouts explored on foot (i.e., with the leg system) as ambulatory spaces. The present research concerns the processes that are used to encode information from these two kinds of space. 
An obvious characteristic of these processes, whether in ambulatory or manipulatory space, is their duration. Whereas visual perception can proceed from a single fixation or a quick scan, the manipulatory and ambulatory systems commonly gather information by a sequence of relatively slow exploratory contact movements over surfaces and along contours. Representation of spatial layout thus imposes heavy demands on memory and temporal-integration processes. It is not surprising, then, to discover that comparisons of two-dimensional spatial perception indicate movement to be much poorer than vision, both for encoding small-scale, manipulatory displays (e.g., Cashdan, 1968; Cleaves \& Royal, 1979; Dodds, Howarth, \& Carter, 1982; Worchel, 1951) and for cognitive mapping of large-scale, ambulatory space (Book \& Garling, 1981; Casey, 1978; Rieser, Lockman, \& Pick, 1980; see also Strelow, 1985, for a general review).

Qualitative differences in the representations achieved with and without vision are equally important (e.g., Brambring, 1976; Casey, 1978; Kerr, 1983; Millar, 1975, 1981). Lederman, Klatzky, and Barber (1985) suggested that representations of space achieved through vision and haptics may differ qualitatively because the processes used by the two modalities are to some extent distinct. They argued that spatial representation through haptics is markedly influenced by cognitive "heuristics," that is, strategic rules that tranform information directly available through the input modality to parameters of the spatial representation. Cognitive heuristics presumably augment more direct perception of spatial layout. ${ }^{1}$ The output of a heuristic rule is integrated with the information acquired from other sources, to determine some composite representation of the spatial display.

Evidence for a variety of spatial-encoding heuristics can be found both with touch and proprioception (Lederman et al., 1985; Millar, 1975, 1981; Richardson, Wuillemin, \& MacKintosh, 1981) and with vision (e.g., Thorndyke, 1981; Tversky, 1981). It seems reasonable to assume that reliance on such heuristics will be greater, the less spatial information is available from more direct sources. This assumption is supported by the finding of greater heuristic influence in visual memory than perception of an explicitly present display (Thorndyke, 1981; Tversky, 1981), in touch as compared with vision (Lederman \& Taylor, 1969), and in visually inexperienced relative to sighted, blindfolded observers (Rieser, Guth, \& Hill, 1982).

Lederman et al. (1985) identified two types of heuristics used to determine spatial parameters of small two-dimensional pathways that were explored manually. In their experiments, blindfolded observers moved an index finger along a raised path from beginning to end, and then evaluated the straight line between the endpoints (which in most cases, was not the same as the pathway that had been felt). When asked about the length of this line, subjects' estimates tended to increase with the length of the pathway, indicating a movement-based heuristic for inferring extent. That is, it appears that greater movement along the pathway tended to increase estimates of the distance between its endpoints. Another type of heuristic emerged when subjects were asked about the orientation of the straight line in space (relative to the table edge). Their estimates tended to err in the direction of inferred natural axes in the plane, which indicated that a spatial-referent heuristic had been used.
The present experiments focus on the use of a movementbased heuristic for estimating distance in movement space. We use the term length distortion to refer to an erroneous increase in estimates of distance as the extent of movement increases. From previous experiments, we know that estimates of straightline extent increase with the amount of movement between the line endpoints. However, those studies did not determine the precise nature of this effect. The length distortion from an irrelevant movement could be mediated by the duration of the movement, by its spatial extent, or by its complexity. (In the Lederman et al., 1985, studies, the pathway direction changed more often for the longer pathways.) One principal purpose of the present studies is to test the hypothesis that length distortion is produced by the duration of movement.

The focus here on the duration hypothesis is motivated by a variety of findings suggesting there can be strong temporal influences on judgments of spatial extent. One is the tau effect (e.g., Schiffman, 1982; Scholtz, 1924), which is a tendency for judgments of the spatial distance between two stimuli to increase with the interstimulus interval. A similar phenomenon is the radial/tangential effect (Wong, 1977): Radial movements from and to the body are judged to be longer than tangential movements of equal extent, which are executed more rapidly. More directly relevant to the present study is research by Wapner, Weinberg, Glick, and Rand (1967), who had subjects judge the relative length of two lines that were passively traced by a finger at different speeds. As would be expected if extent judgments were mediated by movement duration, there was an inverse relation between speed and judged relative length. Finally, note that the influence of duration on distance estimates is so pervasive as to merit common expression in language, as when we say, "Santa Barbara is 90 minutes from L.A." However, this generally arises for fairly large spatial extent, which motivates the second principal purpose of these studies.

Our second concern is whether length distortion due to movement will be manifested in large-scale routes explored through whole-body locomotion, as it does with small-scale pathways that are traced by a finger. If so, the effects obtained in both ambulatory and manipulatory spaces may arise from a common heuristic.

There are few direct comparisons of movement-based encoding of manipulatory and ambulatory spaces. However, comparisons between small- and large-scale spatial encoding are relevant, and these can be found in the broader literature on mental mapping. There appear to be both commonalities and differences in cognitive representation of large- and small-scale displays with any of the foregoing scale criteria. The common elements include (a) the use of landmarks to encode spatial position (Lederman \& Taylor, 1969; Nelson \& Chaiklin, 1980; Sadalla, Staplin, \& Burroughs, 1979), (b) hierarchical organization of spatial units (Allen \& Kirasic, 1985; Maki, 1981; Stevens

\footnotetext{
${ }^{1}$ It is difficult to differentiate between heuristics that are imposed on the output of perception and mechanisms that are intrinsic to perception itself. In fact, the degree to which mediating processes of any kind occur may best be considered as defining a continuum. Because this article considers strategies that appear relatively late and that are often conscious, it seems reasonable to consider these heuristics as secondary to more direct perceptual processes.
} 
\& Coupe, 1978), and (c) basing length judgments on the number of distinct locations in a space (Byrne, 1979; Thorndyke, 1981). As for differences, several studies indicate that the representation of space learned from a map display (one type of smallscale stimulus) is orientation-specific (Evans \& Pezdek, 1980; Levine, Jankovic, \& Palij, 1982), whereas when observers learn about space by navigating through it, judgments appear to be less tied to a particular orientation (Evans \& Pezdek, 1980; Thorndyke \& Hayes-Roth, 1982). The latter effect holds even for blindfolded explorers who consistently navigate a pathway from the same direction throughout learning (Presson \& Hazelrigg, 1984).

The present studies consider similarities in encoding ambulatory and manipulatory routes within the domain of movement space. The experiments factorially vary the speed and extent of movement along a pathway while holding the complexity of the path constant. This design allows us to evaluate several issues. First, if complexity is the mediator of length estimates that produces length distortion, the effect should vanish when simple paths that do not confound complexity and length are used. Second, if spatial extent judgments are not mediated by duration, there should be no effect of speed. But if movement duration is critical, then the estimate of extent should increase as movement speed decreases. In this latter case, the contributions of movement duration and distance can be compared.

\section{Experiment 1: Heuristics for Encoding Manipulatory Space}

\section{Method}

Subjects. A total of 30 students ( 15 of each sex) volunteered to participate as observers. All were normally sighted undergraduate students at Queen's University between ages 19 and 24 years.

Apparatus and stimuli. The stimuli were raised-dot pathways engraved on Brailon plastic $(28 \times 29 \mathrm{~cm})$ with a stylus, creating dots approximately $2 \mathrm{~mm}$ in diameter and $3 \mathrm{~mm}$ apart. Small sandpaper squares marked the endpoints of the path. The distances between pathway endpoints were the same as four used in Lederman et al. (1985): $2.5,4.1,6.7$, and $11.0 \mathrm{~cm}$. These will be called the "euclidean" distance (recognizing that the pathway distance is also a euclidean measure, but along an indirect route). For each euclidean distance, three pathways were created, with pathway distance a factor of 1,3 , or 5 times the euclidean distance. When the multiple was 1 , the pathway was a straight line. When the multiple was 3 , the pathway had a $23^{\circ}$ turn at a point $3 /$ 8 along its length. When the multiple was 5 , the pathway had a $23^{\circ}$ turn at its midpoint. (These values were constrained by the euclidean and pathway lengths. The shapes of the paths can be seen in Figure 3, which shows their large-scale counterparts as they were laid out in a room.) The pathways were presented to the subjects with the (implicit) line between endpoints randomly oriented horizontally, vertically, or at an oblique angle relative to the horizontal table edge.

Procedure. All observers were blindfolded. Practice trials prior to the experimental trials, using both straight and angled paths, initially familiarized the observers with the nature of the pathways and trained them to move their preferred index finger at three distinct speeds. Observers were asked to move across a training pathway at the fastest and slowest speeds with which they were comfortable, and at one speed midway between the two. Several trials were performed at each speed, and feedback about the consistency and distinctiveness of the movement was provided, until the observer established three reasonably distinct ranges of speed. Once observers demonstrated that they could maintain this
Table 1

Actual Durations (in Seconds) Used by Subjects

in Experiments 1 and 2

\begin{tabular}{lccc}
\hline & \multicolumn{3}{c}{ Speed instruction } \\
\cline { 2 - 4 } Condition & Slow & Medium & Fast \\
\hline Experiment 1 & & & \\
$M$ & 20.68 & 9.78 & 1.46 \\
$S D$ & 17.79 & 8.38 & 1.13 \\
Experiment 2 & & & \\
$M$ & 20.06 & 14.00 & 7.82 \\
$S D$ & 17.09 & 11.18 & 6.00 \\
\hline
\end{tabular}

consistency across a series of randomly presented speeds (minimum of nine trials per speed) and pathways, they began the experimental trials.

During each experimental trial, the observer's index finger was first placed at the start of a pathway. Two passes of the stimulus were then performed at an assigned speed. On the first, the observer was to familiarize himself or herself with the pathway at the designated speed. On the second, movement duration was recorded by stopwatch. Immediately after the second pass, observers were asked to estimate either the straight-line distance between the endpoints or the length of the pathway actually explored.

The estimates were made relative to a straight-line standard stimulus of $5.7 \mathrm{~cm}$, fashioned in the same way as the pathways. (This value was chosen to avoid the extremes of the judged values, in keeping with standard psychophysical practice.) Observers felt the standard with their index fingers prior to the experimental trials as much as they deemed necessary for familiarity. Subsequently, the standard was reinstated every five trials. Using whole numbers or fractions, participants made their estimates in terms of the number of standards contained within the judged distance. If the speed on a trial noticeably departed from instruction, the observer was informed, and the trial was presented again later.

The factorial combination of 12 stimuli (4 euclidean distances, 3 pathway lengths in multiples of the euclidean lengths, 3 speed instructions, and 2 judgments) resulted in a total of 72 trials, which were presented in random order in a session lasting approximately $1.5 \mathrm{hr}$. Upon completion of the trials, subjects were asked about the strategies they had used to perform the task.

\section{Results}

Movement speeds. The instructions to move at different speeds resulted in three different durations that differed significantly from one another in an analysis of variance (ANOVA) having one within-subjects factor, $F(2,56)=240.87, p<.0001$. A Newman-Keuls test of the means indicated that they were all significantly different from one another (all $p s<.01$ ). The means and standard deviations of the durations, averaged over all stimuli and both estimates, are shown in Table 1, as are the results of a similar manipulation in Experiment 2.

Pathway estimates. Estimates of the length of the pathway actually traversed are shown by actual pathway length and speed in Figure 1. These estimates were analyzed in an ANOVA with three factors: actual pathway length, speed instruction, and gender. There were no effects involving gender in this or any analysis; thus, no further discussion is merited. There were main effects of pathway length, $F(11,308)=170.2$, and speed, $F(2,56)=23.1$, and a significant Length $\times$ Speed Instruction 


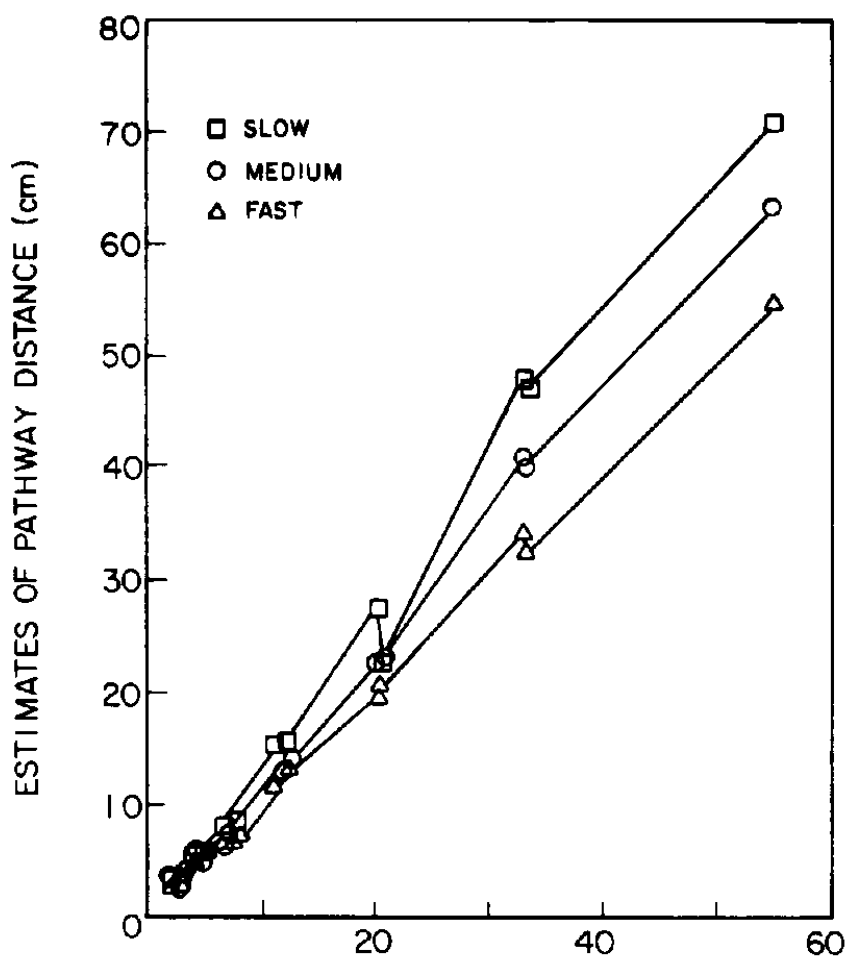

ACTUAL PATHWAY DISTANCE (cm)

Figure 1. Manipulatory space: mean estimates of actual pathway distance (in centimeters) as a function of actual pathway distance (in centimeters) for three speed instructions.

interaction, $F(22,616)=4.8$, all $p s<.001$. As Figure 1 shows, pathway estimates increased directly with the actual value to be estimated, with the rate of increase greatest for the slow movement and least for the fast. In general, estimates were quite accurate: the correlation between pathway estimate and actual value was .98 , and the slope was 1.17 . Thus, overestimation was the rule, which contrasts with the underestimation that predominated in earlier research of Lederman et al. (1985, but with a different response).

Euclidean estimates. The euclidean estimates were subjected to an ANOVA with four factors: gender, pathway distance, true euclidean distance, and speed instruction. All the effects except those involving gender were significant: for pathway, $F(2,56)=51.5$; for euclidean, $F(3,84)=179.2$; for speed, $F(2$, $56)=16.4$; for Pathway $\times$ Euclidean, $F(6,168)=9.4$; for Pathway $\times$ Speed, $F(4,112)=6.9$; for Euclidean $\times$ Speed, $F(6$, $168)=8.2$; and for the three-way interaction, $F(12,336)=3.3$; all ps $<.001$.

The data are shown in Figure 2, which clearly reveals the nature of the effects. There was a general tendency to overestimate the euclidean extent as the pathway factor increased (length distortion). This increased with the euclidean extent being estimated. When the pathway was straight, thus coinciding with the euclidean line, the euclidean estimate was about what would be expected from the slope of the pathway-estimation function (i.e., about 1.17 times the actual distance). There was also an effect of movement speed, such that length distortion was minimal for fast rates of movement and greatest for slow rates. The sum of squares for the euclidean factor was $74.4 \%$ of the total sum of squares attributed to treatment factors. The corresponding values for pathway and speed were $11.1 \%$ and $2.5 \%$.

To further determine the contributions of movement extent and movement duration, correlations were computed between euclidean errors (i.e., estimate minus actual in modulus units) and pathway estimate, and between errors and movement duration. (Note that these factors were not included in the ANOVA, which used actual pathway value in multiple units, and speed instruction.) These correlations were $\mathrm{r}(25)=.90$ for error/pathway estimate and .84 for error/duration (with alpha set at .05 , critical value $=.32$ ). With duration partialed out, the error/ pathway correlation was .77; with pathway estimate partialed out, the error/duration correlation was .59 .

Strategy reports. The reported strategies differed for the two types of estimates. The most commonly reported strategy for euclidean judgments was forming a visual image of the missing leg of the triangle formed by the pathway. For estimating the pathway extent, both visual and temporal strategies were reported. Some subjects estimated the traversal time for the standard at each speed and then divided the pathway movement into corresponding duration units. Alternatively, some subjects reported visualizing the pathway and dividing it into spatial units of the standard's length. Some subjects reported a combination of visual imagery and temporal strategies, resorting to the latter when the pathway became too long to imagine. In general, subjects did not try to use the dots in the pathways as a

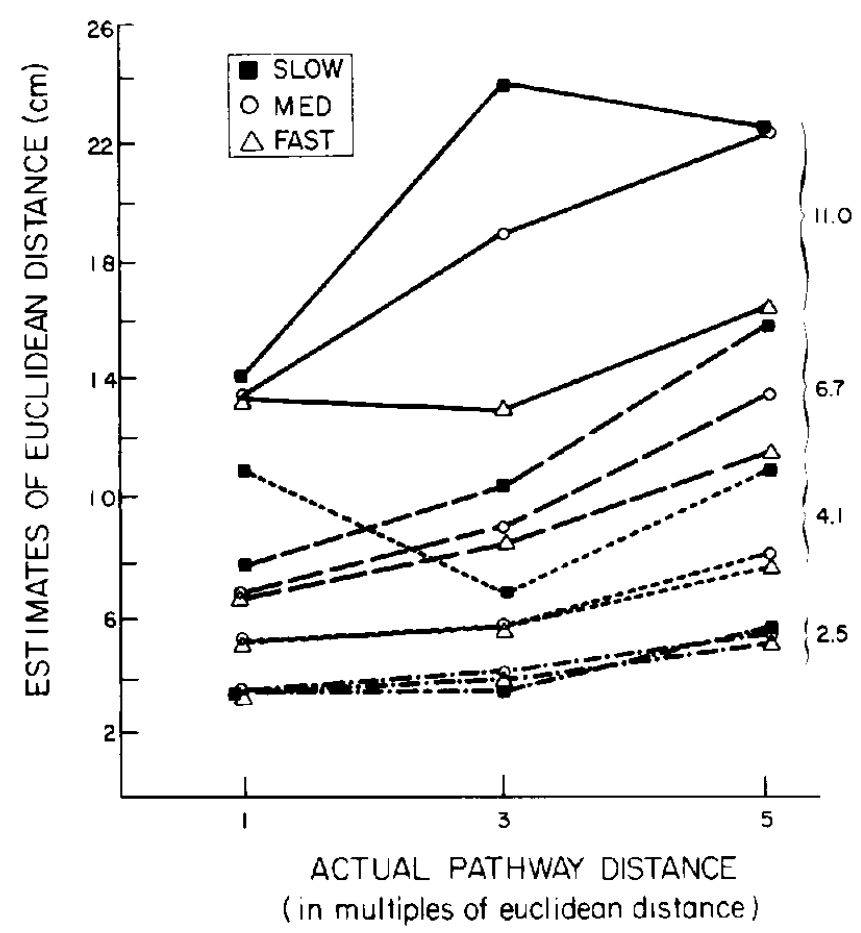

Figure 2. Manipulatory space: mean estimates of euclidean distance (in centimeters) as a function of actual pathway distance in multiples of euclidean distance by actual euclidean distance and speed instruction. 
distance measure, which suggests that they were difficult to resolve and count with a moving finger.

\section{Discussion}

The results of this experiment clearly implicate movement duration as a contributor to manipulatory judgments of extent but secondary to sources of spatial information that do not vary with movement time. The estimates of pathway extent were very strongly related to the actual value, with some contribution from movement time. Similariy, estimates of the inferred euclidean distance between pathway endpoints were directly related to the actual judged value. However, the euclidean estimates were also substantially subject to influences other than the actual distance being estimated; specifically, the pathway extent and movement duration. Of these two influences, the pathway extent was the more potent. In fact, with estimates of the shortest euclidean distances, there was little effect of movement speed, but still an effect of pathway distance.

On the whole, the data suggest that the influences of pathway distance and movement duration were greatest when exploration was extended in time or space. Short, rapid movements led to fairly accurate estimates of distance, whereas long and slow movements produced overestimation errors similar in magnitude to those obtained by Lederman et al. (1985; Note that the previously mentioned underestimation in Lederman et al. was of pathway distances, not euclidean.) This finding supports the general idea that heuristics are used more often when direct spatial apprehension becomes more difficult.

\section{Experiment 2: Heuristics for Encoding Ambulatory Space}

We tum now to the parallel experiment in large-scale, ambulatory space, by which we mean space that surrounds the individual and that must be apprehended through whole-body locomotion (rather than with the hand). In this study, we again varied the speed of a movement as well as its length, and had subjects estimate the extent of either the pathway that was traversed, or the euclidean line between endpoints. The principal questions were, first, whether the length distortion effect would be observed in ambulatory space, and second, whether it woukd be affected by pathway distance and movement duration as in the case of manually explored pathways.

\section{Method}

Subjects. A total of 28 students ( 14 of each sex) from Queen's University, aged $22-35$ years, took part. All were normally sighted with no previous experience in the experimental procedure.

Apparatur and stimudi. Each stimulus was a pathway marked by a handrat of $2.5-\mathrm{cm}$ square metal tubing, mounted $95 \mathrm{~cm}$ in height. The ends of the pathway were expanded with tape as markers. There were a total of nine different pathways, representing three euclidean-distance values $(1,2$, and $4 \mathrm{~m})$ crossed with three pathway lengths $(1,3$, and 5 times euclidean distance). With pathway length of Level 1 , the pathway was the euclidean line. The other pathways changed direction once with an angle of $23^{\circ}$, as in Experiment 1. The pathways were arranged in a room of $12 \mathrm{~m} \times 6 \mathrm{~m}$, as is shown in Figure 3 .

Procedure. All observers were blindfolded prior to entering the experimental room. Practice trials prior to the experimental trials were conducted as in Experiment 1. To establish movement speed, observers first practiced on a 10-m portion of the longest pathway (with angular change) at a show speed, a fast speed, and one halfway in between. Adjustments were made as necessary to establish a difference between fast and slow that allowed for a distinct medium speed and to establish the latter at approximately the midpoint of the extreme speeds. Once three distinct speeds were established on a rough ratio of $1: 2.3$, subjects practiced each until they were consistent within $2 s$ on three consecutive attempts. Transfer to a straight, 5-m path was then tested until subjects succeeded in two passes at each speed within the 2 -s limit. Subjects then traversed the standard to be used as the unit of measurement, a $1.5-\mathrm{m}$ path.

During each experimental trial, the observer first traversed a pathway in one direction at a target speed and was then given feedback about the actual speed. The observer was then timed as he or she retumed along the pathway, and responded on the basis of this second pass. (On switching direction, the observer switched the hand used for self-guidance along the rail.) The experimenter indicated which response was required, a pathway or euclidean estimate, immediately following this second pass.

The estimates were made relative to the $1.5 \cdot \mathrm{m}$ standard, which was traversed prior to the first trial as well as after Trials $5,10,20,35$, and 45 , and upon request. The trials presented the nine pathways, each at three speeds and with each type of estimate, for a total of 54 randomly ordered trials lasting about $2 \mathrm{hr}$. Each pathway was explored beginning at both ends equally often, over conditions and subjects. After completion of the last trial, observers described their estimation strategies verbally.

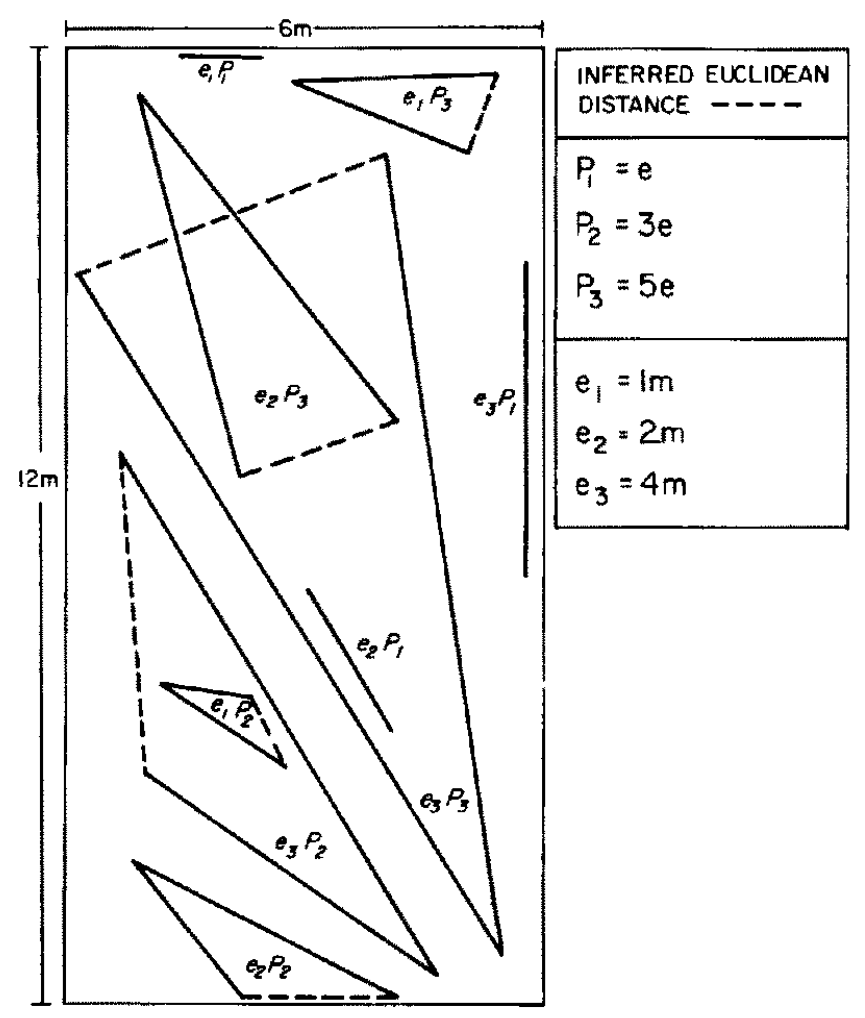

Figure 3. Ambulatory space: experimental layout of the stimulus pathways. 


\section{Results}

Movement speeds. The instructions to move at different speeds resulted in three different durations, the means and standard deviations of which are shown in Table 1, averaged over stimuli and required response. An ANOVA on this within-subjects factor indicated that the speed manipulation was effective $F(2,52)=194.87, p<.0001$. A Newman-Keuls test of the differences between the means indicated that all comparisons were statistically significant (all $p s<.01$ ).

Pathway estimates. These estimates were analyzed with an ANOVA on the factors of pathway distance, speed instruction, and gender. The only significant effect was that of pathway length, $F(8,208)=275.7, p<.001$. No other effect even approached significance. The correlation between pathway estimate and actual pathway distance, $(25)$, was .996 , with a slope of .963 . Thus, these estimates were extremely accurate, as is indicated in Figure 4, which shows the relation between the distance actually moved and the response estimate. The speed instruction factor is not shown here because the functions lie almost on top of one another, the means of the magnitude estimates were $4.69(S D=4.27), 4.94(S D=4.33)$, and 4.74 $(S D=3.90)$ for the low-, medium-, and high-speed instructions, respectively.

Euciddean estimates. An ANOVA on factors actual euclidean value, pathway distance, gender, and speed instruction revealed significant effects of pathway, $F(2,52)=18.7$, and euclidean, $F(2,52)=122.5, p s<.001$. No other effects approached significance. The sum of squares for the euclidean factor was $73.2 \%$ of the total sum of squares for treatment effects. The corresponding value for the pathway factor was $19.2 \%$, and for speed, $0 \%$. The euclidean estimates by actual euclidean value and pathway distance are shown in Figure 5. For reasons given earlier, the effect of speed instruction is not shown; the means of the magnitude estimates of euclidean distance were $2.13(S D=1.95), 2.16(S D=1.88)$, and $2.13(S D=1.71)$, for the low-, medium-, and high-speed instructions, respectively.

The length distortion effect was clearly evident: with euclidean distance constant, estimates increased with pathway length. The magnitude of the effect is comparable to that obtained ear-

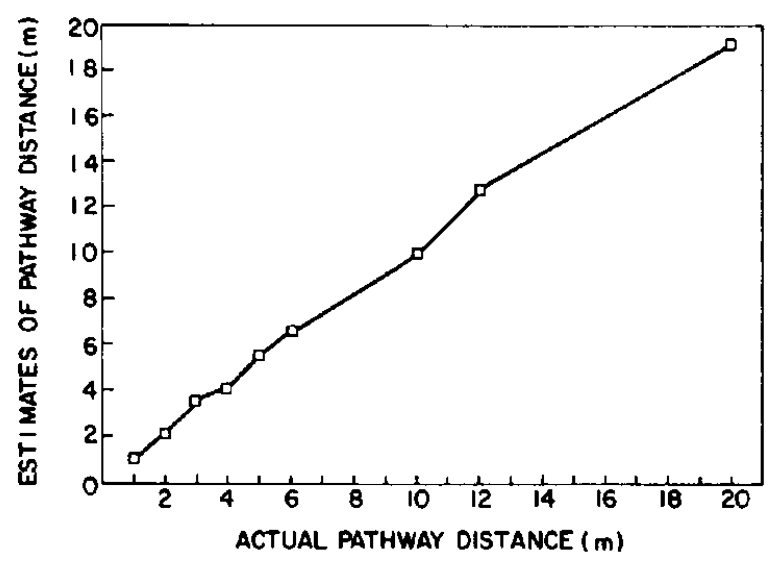

Figure 4. Ambulatory space: Mean estimates of actual pathway distance (in meters) as a function of actual pathway distance in meters.

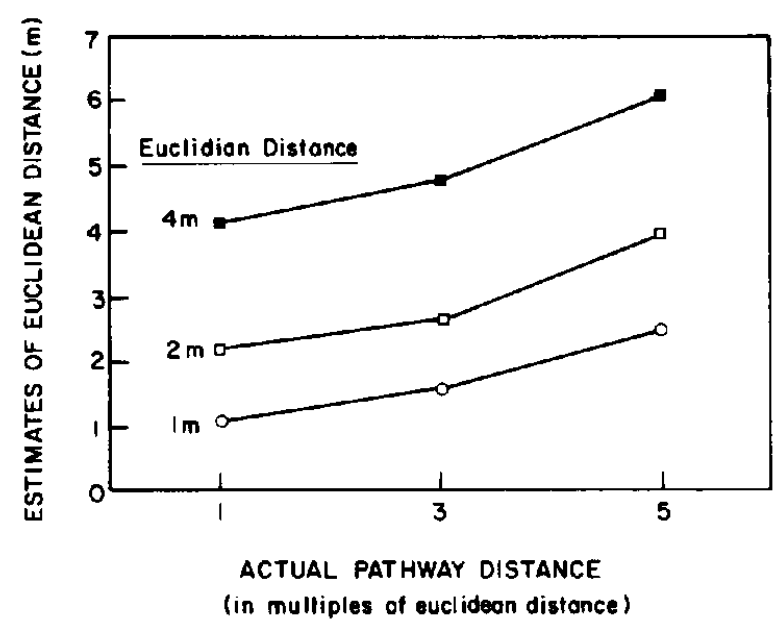

Figture 5. Ambulatory space: Mean estimates of euclidean distance (in meters) as a function of actual pathway distance in multiples of euclidean distance by actual euclidean distance.

lier by Lederman et al. (1985) and that in the present Experiment 1 . There is one difference, however; overestimation of the straight-line pathways was greater in Experiment 1 . As in that study, the euclidean judgments of straight paths produced an estimate that was about what would be expected from the slope of the function for the pathway judgments, that is, error was minimal. Thus, subjects appeared to have treated this condition like a pathway judgment.

The correlation between the euclidean error (estimate minus actual) and the pathway estimate was, $r(25), .72$ (critical value $=.32$ ). With duration partialed out, the correlation was .36. The error/duration correlation was .68 , dropping to .17 with pathway partialed out.

Strategy reports. The post hoc reports revealed two strategies that were used by more than $3 / 4$ of the observers and that depended on the type of estimate. For pathway estimates, observers reported counting steps in the standard and the experimental path and dividing to determine the number of modulus units in the path. Most of the subjects actually determined a step count for the standard at each speed, which would effectively eliminate speed effects. All but one of the observers who did not use this strategy indicated that they thought of it but decided it would be "cheating;" the remaining subject indicated that he attempted to count time. For the euclidean estimates, the dominant strategy, used by all but one observer, was formation of a mental image of the triangle formed by the pathway and then estimation of the third leg (the euclidean path). Most subjects tried to take the angle of the turn into account. Some even kept track of the ratio between the lengths of the two sides (in footsteps) and increased the estimate if the two sides were not equal in length. The one observer who did not report an image strategy said that the estimates were "just guessed."

\section{Discussion}

The results of this study are straightforward: The estimates of pathway extent were very closely related to the actual extent, 
independent of movement duration. The estimates of euclidean extent were strongly affected both by the actual euclidean extent and by the perceived extent of movement along the path (as contrasted with the duration of that movement). That is, length distortion-an increase in the estimate of the inferred euclidean line as the actual pathway length increased-was observed.

\section{General Discussion}

These experiments have considered length distortion in both manipulatory and ambulatory spaces. To repeat, length distortion refers to an erroneous increase in the judged length of spatial extent, as exploratory movement increases. The present studies were concerned with two types of length estimate: that of an actually explored pathway and that of the inferred straight-line distance between its endpoints. In Experiment 1, the pathways were felt by hand; in Experiment 2, they were explored by whole body locomotion. These studies also investigated the role of the duration of movement exploration in length distortion. Specifically, we questioned whether this distortion occurs because of the duration of movements along the path, the spatial extent of those movements, or both. This question was addressed by simultaneously varying the speed of movement as well as its extent.

The results of the two studies are very similar with respect to finding clear evidence of length distortion. In judgments of inferred straight-line distance, movements along the pathway produced comparable magnitudes of distortion in both studies. Such overestimation was primarily restricted to the inferred judgments. The estimates of actual pathway length were quite accurate, although some overestimation was observed in Experiment 1.

The two studies differ markedly, however, with respect to the effects of movement duration. For manipulatory space (Experiment 1), pathway distance and euclidean estimates were affected by duration. But for ambulatory space (Experiment 2), no speed or duration effects were obtained.

The reason for this difference seems clear when we consider the subjects' strategy reports. Subjects in Experiment 2 reported using time-independent heuristics, a footstep metric for estimating the pathway actually traveled and spatial imagery for estimating the inferred line between pathway endpoints. Their reports indicated computing the standard in step units at each speed, counting steps in the pathway, and dividing to compute the pathway estimate in standard units. The pathway-estimation function indicates that this heuristic resulted in highly accurate performance for all three movement speeds. For the euclidean judgments, where a spatial-imagery strategy was reported, the length-distortion effect indicates than any such imagery was erroneously influenced by the pathway extent.

The subjects in Experiment 1 indicated similar strategies. However, by some reports their estimate of the standard was in duration units; in other cases, they reported using more direct spatial estimates. To estimate pathway extent, they could estimate total movement duration or total distance moved, respectively, and divide by standard units. (Alternatively, they could segment the pathway into standard units while traversing it.) Overestimation of pathway extent could reflect underestimating the standard, overestimating the pathway total, or both. Like the ambulatory-space subjects, those in manipulatory space reported using spatial imagery to estimate the euclidean line. It might be thought, therefore, that their strategies were duration independent. In this case, any effects of movement duration on euclidean judgments would be indirect: duration would affect pathway estimates, which would affect the spatial image. However, when the pathway estimate is partialed out, duration still contributes to errors in euclidean judgments. Thus, movement time appears to have a more direct influence on inferred distance estimates than is indicated by the reports.

Had subjects in ambulatory space been prevented from using the footstep strategy, for example, by using instructed vocalization to suppress counting, it seems likely that they would have resembled the manipulatory-space subjects to a greater degree. Under these circumstances, a duration-based strategy would presumably be more compelling, and effects of duration would then be observed. Whether such effects would be greater than the influence of spatial extent is uncertain, but the present data suggest they would not be.

On the whole, these studies make several points regarding the length distortion phenomenon, the role of duration, and the use of heuristics in mapping movement space. They can be summarized as follows.

First, we note that the length distortion effect initially observed with more complex pathways (Lederman et al., 1985) appears to be very robust and general. It is extended here to simple pathways both in manipulatory space explored by the hand and in ambulatory space, where pathways are traversed by foot. The magnitude of the distortion effect was very substantial at both scales, despite the simplicity of the pathways. In fact, the present effect here, with pathways five times the euclidean length, was similar to the increase found by Lederman et al. (1985), where the pathways reached eight times the euclidean length. Thus, there is no indication that complexity is critical to producing length distortion.

As a second point, when the contributions to length distortion of (a) spatial extent and (b) temporal duration of movement are contrasted, our results indicate that spatial extent, in w dependent of movement, is the principal effector. In the study of ambulatory pathways, there were no duration effects, but still there was substantial length distortion. With the manually explored patkways, there were effects of movement duration, on judgments of both pathway extent and inferred straight-line distances. Movements at slower speeds tended to increase overestimation errors, but length distortion was found even when speed effects were minimal, and euclidean judgments were more strongly related to spatial extent than to duration. We can summarize the duration effect by saying that it can (but may not) enhance other, spatially determined, sources of error.

Another contribution of these studies is to underline the use of heuristics in judgments of movement space. In our present and previous studies of manipulatory and ambulatory encoding (Lederman et al., 1985), we have seen evidence for a variety of heuristics: the use of implicit spatial axes as referents, estimates of inferred pathways from spatial extent of actual movement, extent estimates based on duration of movement, the use of knowledge about the geometry of the path, and a footstep metric. One can also infer a variety of representations for spatial information, including imaginal, temporal, and numerical (as 
in the footstep metric). Haptic spatial processing is not unique in the use of heuristics and multiple representations, of course. However, it is arguably more dependent on such devices than the visual modality.

In addition to the theoretical implications of these studies, there are a number of applied issues to which the results are pertinent. We have previously considered one application, tangible graphics for the blind (Lederman et al., 1985). We have argued that to effectively design such displays, we must understand the methods by which they are read. For example, the spatial-referent heuristic suggests adding explicit and nonarbitrary referents to spatial displays. The present findings suggest that even simple displays are subject to inferential processing, which must be considered when materials are constructed for some applications.

A new area of application for this research is in mobility instruction for navigation of ambulatory space by the blind. It is commonly observed that blind persons tend to use a footstep metric; we find here that naive sighted observers gravitate to such a process as well. This suggests that spatial metrics, or temporal metrics adapted to each individual's rate of movement, might be a useful adjunct to navigational displays for the blind. However, it is likely that a footstep metric would prove most useful in cognitively bounded environments, such as a room or building, that require a relatively small number of footsteps to traverse.

Another heuristic that appears to have been used here, reading from a generated image, may not be so directly transferable to a blind population, however, especially those lacking visual experience. That is because the spatial imagery of congenitally blind and sighted, blindfolded observers can be substantially different (Wake, Shimuzu, and Wake, 1980). In a sense, cognitive mapping of movement space is an attempt to create, through movement of body limbs, spatial images that can be translated into action. The lack of success in this area suggests caution in extrapolating the use of images beyond our current subject population.

These reservations aside, we have demonstrated a general phenomenon of length distortion and have traced its underlying causes. In doing so, we have been able to suggest some of the heuristics by which movement space is encoded. These insights may potentially be extended into areas of useful application.

\section{References}

Acredolo, L. (1981). Small- and large-scale spatial concepts in infancy and childhood. In L. Liben, A. Patterson, \& N. Newcombe (Eds.), Spatial representation and behavior across the life span (pp. 63-82). New York: Academic Press.

Allen, G. L., \& Kirasic, K. C. (1985). Effects of cognitive organization of route knowledge on judgments of macrospatial distance. Memory \& Cognition, 13, 218-227.

Book, A., \& Garling, T. (1981). Maintenance of orientation during locomotion in unfamiliar environments. Journal of Experimental Psychology: Human Perception and Performance, 7, 995-1006.

Brambring, M. (1976). The structure of haptic space in the blind and sighted. Psychological Research, 38. 283-302.

Byme, R. (1979). Memory for urban geography. Quarterly Journal of Experimental Psychology, 31, 147-154.

Casey, S. M. (1978) Cognitive mapping by the blind. Journal of Visual Impairment and Blindness, 72, 297-301.
Cashdan, S. (1968). Visual and haptic form discrimination under conditions of successive stimulation. Journal of Experimental Psychology Monographs, 76(2, Pt. 1), 221-224.

Cleaves, W. T, \& Royal, R. W. (1979). Spatial memory for configurations by congenitally blind, late blind, and sighted adults. Journal of Visual Impairment and Blindness, 73, 13-19.

Dodds, A. G., Howarth, C. 1., \& Carter, D. C. (1982). The mental maps of the blind: The role of previous visual experience. Journal of Visual Impairment and Blindness, 76, 5-12.

Evans, G. W., \& Pezdek, K. (1980). Cognitive mapping: Knowledge of real-world distance and location information. Journal of Experimental Psychology: Human Learning and Memory, 6, 13-25.

Garling, T., Book, A., \& Lindberg, E. (1985). Adults's memory representations of the spatial properties of their everyday physical environment. In R. Cohen (Ed.), The development of spatial cognition (pp. 141-184). Hillsdale, NJ: Erlbaum.

Gibson, J. J. (1950). The perception of the visual world. New York: Houghton Mifflin.

Gogel, W. (1978). Size, distance, and depth perception. In M. Friedman \&. E. Carterette (Eds.), Handbook of perception (Vol. 9, pp. 299 -333). New York: Academic Press.

Hirtle, S., \& Jonides, J. (1985). Evidence of hierarchies in cognitive maps. Memory \& Cognition, 13, 208-217.

Ittelson, W. H. (1973). Environment perception and contemporary perceptual theory. In W. H. Ittelson (Ed.), Environment and cognition (pp. 1-19). New York: Seminar Press.

Kerr, N. H. (1983). The role of vision in "visual imagery" experiments: Evidence from the congenitally blind. Journal of Experimental Psy. chology: General, 112, 265-277.

Lederman, S. J., Klatzky, R., \& Barber, P. (1985). Spatial and movement-based heuristics for encoding pattern information through touch. Joumal of Experimental Psychology: General, 114, 33-49.

Lederman, S. J., \& Taylor, M. M. (1969). Perception of interpolated position and orientation by vision and active touch. Perception \& Psychophysics, 6, 153-159.

Levine, M., Jankovic, I. N., \& Palij, M. (1982). Principles of spatial problem solving. Journal of Experimental Psychology: General. 111. 157-175.

Maki, R. H. (1981). Categorization and distance effects with spatial linear orders. Joumal of Experimental Psychology: Human Learning and Memory $7,15-32$

Millar, S. (1975). Spatial memory by blind and sighted children. British Journal of Psychology, 66, 449-459.

Millar, S. (1981). Self-referent and movernent cues in coding spatial location by blind and sighted children. Perception, $10,255-264$.

Moar, I., \& Bower, G. H. (1983). Inconsistency in spatial knowledge. Memory \& Cognition, 11, 107-113

Nelson, T. O., \& Chaiklin, S. (1980) Immediate memory for spatial location. Journal of Experimental Psychology: Human Learning and Memory, 6, 529-545.

Presson, C. C., \& Hazelrigg, M. D. (1984). Building spatial representations through primary and secondary leaming. Journal of Experimental Psychology: Learning, Memory, and Cognition, 10, 716-722.

Richardson, B., Wuillemin, D., \& MacKintosh, G. (1981). Can passive touch be better than active touch? A comparison of active and passive tactile maze learning. British Journal of Psychology, 72, 353-362.

Rieser, J. J., Guth, D., \& Hill, E. (1982). Mental processes mediating independent travel: Implications for orientation and mobility. Journal of Vistul Impairment and Blindness, 76, 213-218.

Rieser, J. J., Lockman, J. J., \& Pick, H. L., Ir. (1980). The role of visual experience in knowledge of spatial layout. Perception \& Psychophysics, 28, 185-190.

Sadalla, E. K., Staplin, L. J., \& Burroughs, W. J. (1979). Retrieval processes in distance cognition. Memory \& Cognition. 7, 291-296. 
Schiffman, H. (1982). Sensation and perception: An integrated approach. Toronto: Wiley.

Scholtz, W. (1924). Experimentelle Untersuchungen uber die phenomenale Grosse von Rounstrecken, die durch Sukzessiv-Darbeitungzweier Reize begrenzt werden. Psychologische Forschung. 5, 219272. (Cited in Jones, B. \& Huang, Y. L., 1982, Space-time dependencies in psychophysical judgment of extent and duration: Algebraic models of the Tau and Kappa effects. Psychological Bulletin. 91, 128142.)

Siegel, A. (1981). The externalization of cognitive maps by children and adults: In search of ways to ask better questions. In L. Liben, A. Patterson, \& N. Newcombe (Eds.), Spatial representation and behavior across the life span (pp. 167-194). New York: Academic Press.

Stevens, A., \& Coupe, P. (1978). Distortions in judged spatial relations. Cognitive Psychology, 10, 422-437.

Strelow, E. (1985). What is needed for a theory of mobility: Direct perception and cognitive maps-Lessons from the blind. Psychological Review, 92, 226-248.

Thorndyke, P. W. (1981). Distance estimation from cognitive maps. Cognitive Psychology, 13, 526-550.
Thorndyke, P., \& Hayes-Roth, B. (1982). Differences in spatial knowledge acquired from maps and navigation. Cognitive Psychology, 14. $560-581$.

Tversky, B. (1981). Distortions in memory for maps. Cognitive Psychology, 13, 407-433.

Wake, T., Shimuzu, Y., \& Wake, H. (1980). Perception of tactile three dimensional information and visual aids for blind persons. Japanese Journal of Ergonomics, 16, 27-36.

Wapner, S., Weinberg, J., Glick, J., \& Rand, G. (1967). Effect of speed of movement on tactual-kinesthetic perception of extent. American Journal of Psychology, 80, 608-613.

Wong, T. S. (1977). Dynamic properties of radial and tangential movements as determinants of the haptic horizontal-vertical illusion with an L-figure. Journal of Experimental Psychology: Human Perception and Performance, 3, 151-164.

Worchel, P. (195I). Space perception and orientation in the blind. Psychological Monographs, 65, (15, Whole No. 332).

Received April 22, 1986

Revision received September 11, 1986

Accepted October 1, 1986 